\title{
Freely indecomposable almost free groups with free abelianization
}

\author{
Samuel M. Corson \\ Communicated by Alexander Yu. Olshanskii
}

\begin{abstract}
For certain uncountable cardinals $\kappa$, we produce a group of cardinality $\kappa$ which is freely indecomposable, strongly $\kappa$-free, and whose abelianization is free abelian of rank $\kappa$. The construction takes place in Gödel's constructible universe $L$. This strengthens an earlier result of Eklof and Mekler.
\end{abstract}

\section{Introduction}

We produce examples of groups which exhibit some properties enjoyed by free groups but which, in other ways, are very far from being free. We recall some definitions before stating the main result. Given a group $G$ and subgroup $H \leq G$, we say $H$ is a free factor of $G$ provided there exists another subgroup $K \leq G$ such that $G=H * K$ is the free product in the natural way (that is, the map $H * K \rightarrow G$ induced by the inclusions of $H$ and $K$ is an isomorphism). We call such a writing $G=H * K$ a free decomposition of $G$ and say that $G$ is freely indecomposable provided there does not exist a free decomposition of $G$ via two nontrivial free factors.

Given a cardinal $\kappa$, we say $G$ is $\kappa$-free if each subgroup of $G$ generated by fewer than $\kappa$ elements is a free group. Historically, a $\kappa$-free group of cardinality $\kappa$ is called almost free [7]. By the theorem of Nielsen and Schreier, every free group is $\kappa$-free for every cardinal $\kappa$. A subgroup $H$ of a $\kappa$-free group $G$ is $\kappa$-pure if $H$ is a free factor of any subgroup $\langle H \cup X\rangle$, where $X \subseteq G$ is of cardinality $<\kappa$. A $\kappa$-free group $G$ is strongly $\kappa$-free provided each subset $X \subseteq G$ with $|X|<\kappa$ is included in a $\kappa$-pure subgroup of $G$ generated by fewer than $\kappa$ elements.

Let ZFC denote the Zermelo-Fraenkel axioms of set theory including the axiom of choice, and let $\mathrm{V}=\mathrm{L}$ denote the assertion that every set is constructible. The theory $\mathrm{ZFC}+\mathrm{V}=\mathrm{L}$ is consistent provided $\mathrm{ZFC}$ is consistent [6]. The set-theoretic 
concepts in the following statement will be reviewed in Section 3, but the reader can, for example, let $\kappa$ be any uncountable successor cardinal (e.g. $\aleph_{1}, \boldsymbol{\aleph}_{2}, \boldsymbol{\aleph}_{\omega+1}$ ).

Theorem 1.1 $(\mathrm{ZFC}+\mathrm{V}=\mathrm{L})$. Let $\kappa$ be an uncountable regular cardinal that is not weakly compact. There exists a group $G$ of cardinality $\kappa$ for which

(1) $G$ is freely indecomposable,

(2) $G$ is strongly $\kappa$-free,

(3) the abelianization $G / G^{\prime}$ is free abelian of rank $\kappa$.

The hypotheses on the cardinal $\kappa$ cannot be dropped since a $\kappa$-free group of cardinality $\kappa$ must be free when $\kappa$ is singular or weakly compact (see respectively [15] and [3]). A group as in the conclusion seems unusual since, on a local level, it is free; on a global level, it is quite unfree and in fact indecomposable, but the abelianization is as decomposable as possible. Theorem 1.1 minus condition (3) was proved in [4], and the construction apparently does not have free abelianization; indeed, the proof that their groups are freely indecomposable involves abelianizing. A non-free $\boldsymbol{\aleph}_{1}$-free group of cardinality $\boldsymbol{\aleph}_{1}$ which abelianizes to a free abelian group was produced by Bitton [1] using only ZFC, and Theorem 1.1 can be considered a constructible universe strengthening of his result. The first construction of a non-free almost free group of cardinality $\aleph_{1}$ was given by Higman [7] without any extra set-theoretic assumptions; a strongly $\boldsymbol{\aleph}_{1}$-free group of cardinality $\aleph_{1}$ produced only from ZFC was given by Mekler [13]. The reader can find other results related to almost free (abelian) groups in such works as $[2,5,12]$.

We note that it is not possible to produce a group $G$ of cardinality $\geq \kappa$ whose every subgroup of cardinality $\kappa$ satisfies conditions (1)-(3) of Theorem 1.1. This is because, for every uncountable locally free group $G$, there exists a free subgroup $H \leq G$ with $|H|=|G|$ (see [14, Theorem 1.1]). Finally, we mention that the construction used in proving Theorem 1.1 allows one, as in [4], to construct $2^{\kappa}$ many pairwise non-isomorphic groups of this description using [16].

\section{Some group-theoretic lemmas}

The following appears as [7, Lemma 1].

Lemma 2.1. If $K$ is a free factor of $G$ and $H$ is a subgroup of $G$, then $H \cap K$ is a free factor of $H$.

We will use the following construction in our proof of Theorem 1.1. 
Construction 2.2. Suppose that we have a free group $F_{a}$ with free decomposition $F_{a}=F_{0} * F_{1} * F_{2, a}$ with $F_{1}$ nontrivial and $F_{2, a}$ freely generated by $\left\{t_{n}\right\}_{n \in \omega}$. Let $F_{b}$ be a free group with free decomposition $F_{b}=F_{0} * F_{1} * F_{2, b}$, where $F_{2, b}$ is freely generated by $\left\{z_{n}\right\}_{n \in \omega}$. Let $y$ be an element of a free generating set for $F_{1}$. Define $\phi: F_{a} \rightarrow F_{b}$ so that $\phi \uparrow F_{0} * F_{1}$ is the identity and $\phi\left(t_{n}\right)=y z_{n+1}^{-1} z_{n} z_{n+1}$.

Property (iv) of the following lemma compares to [1, Lemma 2.9 (3) \& (4)].

Lemma 2.3. Let $F_{2, a, n}=\left\langle t_{0}, \ldots, t_{n-1}\right\rangle$. The map $\phi$ satisfies the following:

(i) $\phi$ is a monomorphism;

(ii) $z_{n} \notin \phi\left(F_{a}\right)$ for all $n \in \omega$;

(iii) $\phi\left(F_{a}\right)$ is not a free factor of $F_{b}$, but $\phi\left(F_{0} * F_{1} * F_{2, a, n}\right)$ is a free factor for every $n \in \omega$;

(iv) the equality $\left(\phi\left(F_{a}\right)\right)^{\prime}=\phi\left(F_{a}\right) \cap F_{b}^{\prime}$ holds, and the natural induced map $\bar{\phi}: F_{a} / F_{a}^{\prime} \rightarrow F_{b} / F_{b}^{\prime}$ is an isomorphism.

Proof. Fix a possibly empty set of free generators $X$ for $F_{0}$ and a possibly empty set $Y$ such that the disjoint union $Y \sqcup\{y\}$ freely generates $F_{1}$. Now $\phi\left(F_{a}\right)$ is the subgroup

$$
\left\langle X \cup Y \cup\{y\} \cup\left\{y z_{n+1}^{-1} z_{n} z_{n+1}\right\}_{n \in \omega}\right\rangle=\left\langle X \cup Y \cup\{y\} \cup\left\{z_{n+1}^{-1} z_{n} z_{n+1}\right\}_{n \in \omega}\right\rangle,
$$

and the generators $X \cup Y \cup\{y\} \cup\left\{z_{n+1}^{-1} z_{n} z_{n+1}\right\}_{n \in \omega}$ freely generate $\phi\left(F_{a}\right)$ since they satisfy the Nielsen property (see [11, I.2] and [8, Example 2.8 (iii)]). The set $X \cup Y \cup\{y\} \cup\left\{y^{-1} t_{n}\right\}_{n \in \omega}$ is a free generating set for $F_{a}$ and maps bijectively under $\phi$ to the free generating set $X \cup Y \cup\{y\} \cup\left\{z_{n+1}^{-1} z_{n} z_{n+1}\right\}_{n \in \omega}$ for $\phi\left(F_{a}\right)$. Thus $\phi$ is a monomorphism, and we have (i). Moreover, since

$$
X \cup Y \cup\{y\} \cup\left\{z_{n+1}^{-1} z_{n} z_{n+1}\right\}_{n \in \omega}
$$

satisfies the Nielsen property, it is clear that $z_{n} \notin \phi\left(F_{a}\right)$ for all $n \in \omega$, and we have (ii).

For (iii), we notice that the smallest normal subgroup in $F_{b}$ including $\phi\left(F_{a}\right)$, which we denote $\left\langle\left\langle\phi\left(F_{a}\right)\right\rangle\right\rangle$, is precisely $F_{b}$ since the free generators listed for $F_{b}$ are conjugate in $F_{b}$ to free generators of $\phi\left(F_{a}\right)$. Since $\phi\left(F_{a}\right)$ is a proper subgroup of $F_{b}$, this means that $\phi\left(F_{a}\right)$ cannot be a free factor of $F_{b}$. On the other hand, we have $\phi\left(F_{0} * F_{1} * F_{2, a, n}\right)$ generated by

$$
X \cup Y \cup\{y\} \cup\left\{y z_{1}^{-1} z_{0} z_{1}, \ldots, y z_{n}^{-1} z_{n-1} z_{n}\right\},
$$

and we claim that $X \cup Y \cup\{y\} \cup\left\{y z_{1}^{-1} z_{0} z_{1}, \ldots, y z_{n}^{-1} z_{n-1} z_{n}\right\} \cup\left\{z_{n}, z_{n+1}, \ldots\right\}$ is a free generating set for $F_{b}$. It is plain that

$$
\left\langle\{y\} \cup\left\{y z_{1}^{-1} z_{0} z_{1}, \ldots, y z_{n}^{-1} z_{n-1} z_{n}\right\} \cup\left\{z_{n}\right\}\right\rangle=\left\langle\{y\} \cup\left\{z_{0}, \ldots, z_{n}\right\}\right\rangle,
$$


and since finitely generated free groups are Hopfian, we get that

$$
\{y\} \cup\left\{y z_{1}^{-1} z_{0} z_{1}, \ldots, y z_{n}^{-1} z_{n-1} z_{n}\right\} \cup\left\{z_{n}\right\}
$$

is a free generating set of the free factor $\left\langle\{y\} \cup\left\{z_{0}, \ldots, z_{n}\right\}\right\rangle$ of $F_{b}$. Thus, indeed, $X \cup Y \cup\{y\} \cup\left\{y z_{1}^{-1} z_{0} z_{1}, \ldots, y z_{n}^{-1} z_{n-1} z_{n}\right\} \cup\left\{z_{n}, z_{n+1}, \ldots\right\}$ is a free generating set for $F_{b}$, and so

$$
\phi\left(F_{0} * F_{1} * F_{2, a, n}\right)=\left\langle X \cup Y \cup\{y\} \cup\left\{y z_{1}^{-1} z_{0} z_{1}, \ldots, y z_{n}^{-1} z_{n-1} z_{n}\right\}\right\rangle
$$

is a free factor of $F_{b}$ and we have shown (iii).

For condition (iv), certainly the inclusion $\left(\phi\left(F_{a}\right)\right)^{\prime} \subseteq \phi\left(F_{a}\right) \cap F_{b}^{\prime}$ holds. Moreover, a word $w$ in $\left(X \cup Y \cup\{y\} \cup\left\{z_{n}\right\}_{n \in \omega}\right)^{ \pm 1}$ represents an element of $F_{b}^{\prime}$ if and only if the sum of the exponents of each element in $X \cup Y \cup\{y\} \cup\left\{z_{n}\right\}_{n \in \omega}$ is 0 . By treating each element of $\left\{z_{n+1}^{-1} z_{n} z_{n+1}\right\}_{n \in \omega}$ as an unreducing letter, a word in

$$
\left(X \cup Y \cup\{y\} \cup\left\{z_{n+1}^{-1} z_{n} z_{n+1}\right\}_{n \in \omega}\right)^{ \pm 1}
$$

represents an element of $\left(\phi\left(F_{a}\right)\right)^{\prime}$ if and only if the sum of the exponents of each element of $X \cup Y \cup\{y\} \cup\left\{z_{n+1}^{-1} z_{n} z_{n+1}\right\}_{n \in \omega}$ is 0 . This is clearly equivalent to having the sum of the exponents of each letter in $X \cup Y \cup\{y\} \cup\left\{z_{n}\right\}_{n \in \omega}$ be 0 , so we have $\left(\phi\left(F_{a}\right)\right)^{\prime}=\phi\left(F_{a}\right) \cap F_{b}^{\prime}$. Thus the map $\bar{\phi}: F_{a} / F_{a}^{\prime} \rightarrow F_{b} / F_{b}^{\prime}$ is injective. Moreover, since each element of $X \cup Y \cup\{y\} \cup\left\{z_{n}\right\}_{n \in \omega}$ is conjugate in $F_{b}$ to an element of $X \cup Y \cup\{y\} \cup\left\{z_{n+1}^{-1} z_{n} z_{n+1}\right\}_{n \in \omega}$, the map $\bar{\phi}$ is onto as well.

We recall some notions for free products of groups (see [11, Section IV.1]). Suppose that we have a free product $L_{0} * L_{1}$. We call the nontrivial elements in $L_{0} \cup L_{1}$ letters. Each element $g \in L_{0} * L_{1}$ can be expressed uniquely as a product of letters $g=h_{0} h_{1} \cdots h_{n-1}$ such that $h_{i} \in L_{0}$ if and only if $h_{i+1} \in L_{1}$ for all $0 \leq i<n-1$ (this is the reduced or normal form of the element). We call the number $n$ the length of $g$, denoted $\operatorname{Len}(g)$. Thus the identity element has length 0 and a nontrivial element has length 1 if and only if it is a letter. Given a writing of an element of $L_{0} * L_{1}$ as a product of nontrivial elements $h_{0} \cdots h_{n-1}$ in $L_{0} \cup L_{1}$, it is easy to determine the normal form of the element by taking a consecutive pair $h_{i} h_{i+1}$ for which $h_{i}, h_{i+1}$ are both in $L_{0}$ or both in $L_{1}$ and performing the group multiplication in the appropriate group. This either gives the trivial element, in which case we remove the pair $h_{i} h_{i+1}$ from the expression, or it gives a nontrivial element $g_{i}$, and we replace $h_{i} h_{i+1}$ with $g_{i}$ in the expression. This process reduces the number of letters in the writing by at least 1 every time, and so the process must eventually terminate, and it terminates at the normal form. We will generally consider an element of $L_{0} * L_{1}$ as a word (the normal form) in the letters. For 
words $w_{0}$ and $w_{1}$ in the letters, we will use $w_{0} \equiv w_{1}$ to represent that $w_{0}$ and $w_{1}$ are the same word letter-for-letter when read from left to right.

We say an element of $L_{0} * L_{1}$ is cyclically reduced if its reduced form is either of length 0 or 1 or begins with a letter in $L_{j}$ and ends with a letter in $L_{1-j}$. A cyclically reduced element is of minimal length in its conjugacy class, and if two cyclically reduced elements are conjugate to each other, then the normal form of one is a cyclic shift of the other ( $w$ is a cyclic shift of $u$ if we can write $w$ as a concatenation $w \equiv v_{0} v_{1}$ such that $u \equiv v_{1} v_{0}$ ). Each element $g$ of $L_{0} * L_{1}$ is conjugate to a cyclically reduced element $h$, and we call $\operatorname{Len}(h)$ the cyclic length of $g$.

Lemma 2.4. If $\psi: F_{a} \rightarrow L_{0} * L_{1}$ is a monomorphism such that

$$
\psi\left(F_{1}\right)=\psi\left(F_{a}\right) \cap L_{0} \quad \text { and } \quad \psi\left(F_{0} * F_{2, a}\right)=\psi\left(F_{a}\right) \cap L_{1},
$$

then there does not exist a monomorphism $\theta: F_{b} \rightarrow L_{0} * L_{1}$ for which $\theta \circ \phi=\psi$.

Proof. Suppose on the contrary that such a $\theta$ exists. We will treat $F_{a}$ as a subgroup of $F_{b}$ since $\phi$ is a monomorphism and treat $F_{a}$ and $F_{b}$ as subgroups of $L_{0} * L_{1}$ such that $F_{1}=F_{a} \cap L_{0}$ and $F_{0} * F_{2, a}=F_{a} \cap L_{1}$. We have $t_{n}=y z_{n+1}^{-1} z_{n} z_{n+1}$ for all $n \in \omega$, and so $y^{-1} t_{n}=z_{n+1}^{-1} z_{n} z_{n+1}$. Since $y^{-1} \in L_{0}$ and $t_{n} \in L_{1}$, we see that $y^{-1} t_{n}$ is cyclically reduced and $\operatorname{Len}\left(y^{-1} t_{n}\right)=2$. Therefore, for each $n \in \omega$, we know $z_{n}$ is of cyclic length 2 , so $\operatorname{Len}\left(z_{n}\right) \geq 2$, and any cyclic reduction of $z_{n}$ must be a cyclic shift of $y^{-1} t_{n}$. We claim that $\operatorname{Len}\left(z_{n}\right) \geq \operatorname{Len}\left(z_{n+1}\right)+1$ for all $n \in \omega$. This immediately gives $\operatorname{Len}\left(z_{0}\right) \geq \operatorname{Len}\left(z_{n}\right)+n$ for all $n \in \omega$, which is a contradiction.

It remains to prove that $\operatorname{Len}\left(z_{n}\right) \geq \operatorname{Len}\left(z_{n+1}\right)+1$. To economize on writing subscripts, we will show that $\operatorname{Len}\left(z_{0}\right) \geq \operatorname{Len}\left(z_{1}\right)+1$, and the same proof will work for general $n$ by adding $n$ to the subscripts of $t_{0}, z_{0}, t_{1}, z_{1}$. Suppose to the contrary that $\operatorname{Len}\left(z_{0}\right) \leq \operatorname{Len}\left(z_{1}\right)$. We have $z_{1} y^{-1} t_{0} z_{1}^{-1}=z_{0}$. Since $z_{1}$ is nontrivial, it must end with a letter of $L_{0}$ or a letter of $L_{1}$.

Case A: $z_{1}$ ends with a letter of $L_{0}$. In this case, it must be that $z_{1}$ ends with $y$ since otherwise we readily see from the reduced form for $z_{0}=z_{1} y^{-1} t_{0} z_{1}^{-1}$ that $\operatorname{Len}\left(z_{0}\right)=\left(2 \operatorname{Len}\left(z_{1}\right)+2\right)-1=2 \operatorname{Len}\left(z_{1}\right)+1>\operatorname{Len}\left(z_{1}\right)$ contrary to our assumption that $\operatorname{Len}\left(z_{0}\right) \leq \operatorname{Len}\left(z_{1}\right)$. Also, the second-to-last letter of $z_{1}$ must be $t_{0}^{-1}$ since otherwise $L\left(z_{0}\right)=\left(2 \operatorname{Len}\left(z_{1}\right)+2\right)-3=2 \operatorname{Len}\left(z_{1}\right)-1>\operatorname{Len}\left(z_{1}\right)$ since $\operatorname{Len}\left(z_{1}\right) \geq 2$. Thus we may write $z_{1}$ as a reduced word $z_{1}=w\left(t_{0}^{-1} y\right)^{k}$ with $k \geq 1$ and maximal. Notice that $w$ is nonempty, for otherwise $z_{1}=\left(t_{0}^{-1} y\right)^{k}$ and $z_{0}=y^{-1} t_{0}$, and therefore $z_{0}$ and $z_{1}$ commute instead of generating a free subgroup of rank 2. The word $w$ must end with a letter from $L_{0}$ since $w\left(t_{0}^{-1} y\right)^{k}$ is reduced. Moreover, $\operatorname{Len}(w) \geq 2$ since otherwise $w=g \in L_{0}$ and $z_{1}$ is conjugate 
to $\left(t_{0}^{-1} y\right)^{k-1} t_{0}^{-1} y g$, and cyclically reducing this word cannot produce a cyclic shift of $y^{-1} t_{1}$. Then the second-to-last letter of $w$ is an element from $L_{1}$. If the last letter of $w$ is $y$, then the second-to-last letter of $w$ is not $t_{0}^{-1}$ by maximality of $k$, and we get

$$
\operatorname{Len}\left(z_{0}\right)=\left(2 \operatorname{Len}\left(z_{1}\right)+2\right)-4 k-2-1=2 \operatorname{Len}\left(z_{1}\right)-1-4 k .
$$

If, on the other hand, the last letter of $w$ is $g \in L_{0} \backslash\{1, y\}$, then we see that

$$
\operatorname{Len}\left(z_{0}\right)=\left(2 \operatorname{Len}\left(z_{1}\right)+2\right)-4 k-1=2 \operatorname{Len}\left(z_{1}\right)-4 k+1,
$$

so, in either case, we know $\operatorname{Len}\left(z_{0}\right) \geq 2 \operatorname{Len}\left(z_{1}\right)-1-4 k$. Since we are assuming $\operatorname{Len}\left(z_{1}\right) \geq \operatorname{Len}\left(z_{0}\right)$, we have $\operatorname{Len}\left(z_{1}\right) \leq 4 k+1$.

Write $z_{1}=\left(y^{-1} t_{0}\right)^{m} u\left(t_{0}^{-1} y\right)^{k}$ with $m \geq 0$ maximal. Certainly, $u$ is nontrivial since otherwise $z_{1}$ would commute with $z_{0}$. We also claim that $u$ must begin and end with a letter from $L_{0}$. It is clear, since $z_{1}$ is written in reduced form, that $u$ must end with a letter from $L_{0}$ since $t_{0}^{-1} \in L_{1}$. Also, if $m>0$, we have $u$ beginning with a letter from $L_{0}$ for a similar reason. But if $m=0$, then we have $z_{1}=u\left(t_{0}^{-1} y\right)^{k}$, and were it the case that $u$ started with a letter in $L_{1}$, then this writing would already be cyclically reduced and $z_{1}$ would have cyclic length greater than 2 since $u$ is nontrivial. Thus, in all cases, $u$ must begin and end with a letter from $L_{0}$.

If $m>k$, then $\operatorname{Len}\left(z_{1}\right)=2 m+2 k+\operatorname{Len}(u)>4 k+1$. If $k=m$, then, since $4 k+1 \geq \operatorname{Len}\left(z_{1}\right)=2 m+2 k+\operatorname{Len}(u)=4 k+\operatorname{Len}(u)$, we get $\operatorname{Len}(u)=1$. Then $z_{1}$ is conjugate to an element of length 1 , contradicting the fact that $z_{1}$ is of cyclic length 2 . Thus $k>m$.

If $k>m+1$, then, by maximality of $m$, we conjugate $z_{1}$ to a word

$$
u\left(t_{0}^{-1} y\right)^{k-m-1} t_{0}^{-1} y,
$$

where $u$ might or might not start with $y^{-1}$, but if it starts with $y^{-1}$, the second letter of $u$ would not be $t_{0}$. Thus, whether or not $u$ starts with $y^{-1}$, we know that $u\left(t_{0}^{-1} y\right)^{k-m-1} t_{0}^{-1} y$ has cyclic length at least 3 despite being conjugate to $z_{1}$, a contradiction. Thus we know precisely that $k=m+1$, and so $z_{1}$ is conjugate to $u t_{0}^{-1} y$. If $u$ does start with $y^{-1}$, say $u \equiv y^{-1} v$ for some word $v$, then we get $z_{1}$ conjugate to $v t_{0}^{-1}$. Since the cyclic length of $z_{1}$ is 2 , we know that $v$ is nonempty, must start with a letter from $L_{1}$ and that letter must not be $t_{0}$ by the maximality of $m$. Then $v \equiv t v_{0}$ with $t \in L_{1} \backslash\left\{1, t_{0}^{-1}\right\}$, and $v_{0}$ begins and ends with a letter in $L_{0}$. Thus $z_{1}$ is conjugate to $v_{0}\left(t_{0}^{-1} t\right)$ which is a cyclically reduced word. Since $z_{1}$ has cyclic length 2 and cyclic reduction $y^{-1} t_{1}$, we obtain that $v_{0}=y^{-1}$ and $t_{0}^{-1} t=t_{1}$. But now

$$
z_{1}=\left(y^{-1} t_{0}\right)^{k-1} u\left(t_{0}^{-1} y\right)^{k}=\left(y^{-1} t_{0}\right)^{k-1} y^{-1}\left(t_{0} t_{1}\right) y^{-1}\left(t_{0}^{-1} y\right)^{k}
$$


so that $z_{1}$ is expressed as a product of elements in $F_{a}$, contradicting Lemma 2.3 part (ii). Therefore, $u$ must start with some $g \in L_{0} \backslash\left\{1, y^{-1}\right\}$, say $y \equiv g v_{0}$. Now we conjugate $u t_{0}^{-1} y \equiv g v_{0} t_{0}^{-1} y$ to $v_{0} t_{0}^{-1}(y g)$ which is cyclically reduced (by considering $(y g)$ as a single letter in $\left.L_{0}\right)$. But $v_{0} t_{0}^{-1}(y g)$ cannot possibly be a cyclic shift of $y^{-1} t_{1}$, regardless of whether $v_{0}$ is empty or not. This finishes the proof of Case A.

Case B: $z_{1}$ ends with a letter of $L_{1}$. The reasoning in this case follows that in the other case more or less, and we give the sketch. Arguing as before, we see that $z_{1}$ must end with $t_{0}$, and the second-to-last letter must be $y^{-1}$. Write $z_{1} \equiv w\left(y^{-1} t_{0}\right)^{k}$ with $k \geq 1$ maximal. As before, $w$ is nonempty. Again, we have $\operatorname{Len}\left(z_{0}\right) \geq 2 \operatorname{Len}\left(z_{1}\right)-1-4 k$ from which $\operatorname{Len}\left(z_{1}\right) \leq 4 k+1$. Writing

$$
z_{1} \equiv\left(t_{0}^{-1} y\right)^{m} u\left(y^{-1} t_{0}\right)^{k}
$$

with $m \geq 0$ maximal, we again see that $u$ is nonempty and that $k=m+1$. Also, $u$ must begin and end with letters from $L_{1}$. Therefore, $z_{1}$ is conjugate to $u y^{-1} t_{0}$. If $u \equiv t_{0}^{-1} v$ for some $v$, then we get $z_{1}$ conjugate to $v y^{-1}$, and as $m$ was maximal, the word $v$ starts with an element of $L_{0}$ which is not $y$. Then $v \equiv g v_{0}$ with $g \in L_{0} \backslash\{1, y\}$ and $v_{0}$ nontrivial since $u$, and therefore $v$, must end in a letter from $L_{1}$. Conjugating $v y^{-1}$ to the cyclically reduced word $v_{0}\left(y^{-1} g\right)$, we must have that this word is a cyclic shift of $y^{-1} t_{1}$. Then $v_{0}=t_{1}$, but $y^{-1} g$ cannot be $y^{-1}$ since $g$ was nontrivial. Therefore, it must be that $u \equiv t v$ for some $t \in L_{1} \backslash\left\{1, t_{0}\right\}$. Conjugating $u y^{-1} t_{0}=t v y^{-1} t_{0}$ to the cyclically reduced word $v y^{-1}\left(t_{0} t\right)$, it must be that this word is a cyclic shift of $y^{-1} t_{1}$. Then $v$ is empty and $t_{0} t=t_{1}$. Therefore,

$$
z_{1}=\left(t_{0}^{-1} y\right)^{k-1} u\left(y^{-1} t_{0}\right)^{k}=\left(t_{0}^{-1} y\right)^{k-1}\left(t_{0}^{-1} t_{1}\right)\left(y^{-1} t_{0}\right)^{k},
$$

and we have $z_{1} \in F_{a}$, which contradicts Lemma 2.3 part (ii). This completes the proof of Case B and of the lemma.

\section{Proof of the main theorem}

The groups that we produce for Theorem 1.1 will follow the induction used in [4, Theorem 2.2], using Construction 2.2 at the key stages. We will use combinatorial principles which follow from $\mathrm{ZFC}+\mathrm{V}=\mathrm{L}$ to rule out any possible free decomposition while ensuring strong $\kappa$-freeness and free abelianization. We first review some concepts from set theory.

Definitions 3.1. (see [9]) Recall that a cardinal number is naturally considered as an ordinal number which cannot be injected into a proper initial subinterval of itself. A subset $E$ of an ordinal $\alpha$ is bounded in $\alpha$ if there exists $\beta<\alpha$ which 
is an upper bound on $E$. The cofinality of an ordinal $\alpha$ is the least cardinal $\kappa$ for which there exists an unbounded $E \subseteq \alpha$ of cardinality $\kappa$. An infinite cardinal $\kappa$ is regular if the cofinality of $\kappa$ is $\kappa$. A subset $C$ of ordinal $\alpha$ is club if it is unbounded in $\alpha$ and closed under the order topology in $\alpha$. The intersection of two club sets in an uncountable regular cardinal is again a club set. A subset $E$ of ordinal $\alpha$ is stationary if it has nonempty intersection with every club subset of $\alpha$. The intersection of a club set and a stationary set in a regular cardinal is again stationary. We mention that weakly compact cardinals are inaccessible, and it is therefore consistent to assume that a universe of set theory does not contain any (see $[9$, Chapters 9, 17]).

The following is a theorem of Solovay (see [16] or [9, Theorem 8.10]).

Theorem 3.2. If $\kappa$ is an uncountable regular cardinal, then each stationary subset of $\kappa$ can be decomposed as the disjoint union of $\kappa$ many stationary subsets of $\kappa$.

We quote Jensen's $\diamond_{K}(E)$ principle ([10, Lemma 6.5] or [9, Assertion 27.16]), remark an easy consequence (see [4, page 97]) and quote one more result of Jensen (see [10, Theorem 5.1], [4, Theorem 1.3]).

Theorem 3.3 $(\mathrm{ZFC}+\mathrm{V}=\mathrm{L})$. If $\kappa$ is an uncountable regular cardinal and $E \subseteq \kappa$ is stationary in $\kappa$, there exists a sequence $\left\{S_{\alpha}\right\}_{\alpha \in E}$ such that $S_{\alpha} \subseteq \alpha$ and, for any $J \subseteq \kappa$, the set $\left\{\alpha \in E \mid J \cap \alpha=S_{\alpha}\right\}$ is stationary in $\kappa$.

Remark 3.4. From a sequence given by Theorem 3.3, one obtains a sequence of ordered pairs $\left\{\left(T_{\alpha}^{0}, T_{\alpha}^{1}\right)\right\}_{\alpha \in E}$ for which $T_{\alpha}^{0}, T_{\alpha}^{1} \subseteq \alpha$ and, given any subsets $A, B \subseteq \kappa$, the set $\left\{\alpha \in E \mid A \cap \alpha=T_{\alpha}^{0}\right.$ and $\left.B \cap \alpha=T_{\alpha}^{1}\right\}$ is stationary in $\kappa$. To see this, we give the product $\{0,1\} \times \kappa$ the lexicographic order. There is an order isomorphism $f:\{0,1\} \times \kappa \rightarrow \kappa$ under which $f(0, \alpha)=\alpha$ for every limit ordinal $\alpha<\kappa$. Let $f_{i}: \kappa \rightarrow \kappa$ be given by $f_{i}(\alpha)=f(i, \alpha)$ for each $i \in\{0,1\}$, so $f_{0}$ has disjoint image from $f_{1}$. Notice that, for each limit ordinal $\alpha<\kappa$ and $X \subseteq \kappa$, we have $f_{i}(X \cap \alpha)=f_{i}(X) \cap \alpha$. Letting $E_{0}$ be the intersection of $E$ with the set of limit ordinals below $\kappa$, we have that $E_{0}$ is stationary as the intersection of a stationary with a club. For $\alpha \in E_{0}$, define $T_{\alpha}^{0}=f_{0}^{-1}\left(S_{\alpha}\right)$ and $T_{\alpha}^{1}=f_{1}^{-1}\left(S_{\alpha}\right)$. Given $A, B \subseteq \kappa$, we let $J=f_{0}(A) \sqcup f_{1}(B)$ and notice that

$$
\begin{aligned}
\{\alpha \in & \left.E_{0} \mid A \cap \alpha=T_{\alpha}^{0} \text { and } B \cap \alpha=T_{\alpha}^{1}\right\} \\
& =\left\{\alpha \in E_{0} \mid f_{0}(A \cap \alpha) \sqcup f_{1}(B \cap \alpha)=S_{\alpha}\right\} \\
& =\left\{\alpha \in E_{0} \mid\left(f_{0}(A) \cap \alpha\right) \sqcup\left(f_{1}(B) \cap \alpha\right)=S_{\alpha}\right\} \\
& =\left\{\alpha \in E_{0} \mid J \cap \alpha=S_{\alpha}\right\}
\end{aligned}
$$


is stationary in $\kappa$. Letting $T_{\alpha}^{0}=\emptyset=T_{\alpha}^{1}$ for $\alpha \in E \backslash E_{0}$ gives the desired sequence of ordered pairs.

Theorem 3.5 $(\mathrm{ZFC}+\mathrm{V}=\mathrm{L})$. If $\kappa$ is an uncountable regular cardinal which is not weakly compact, then there is a stationary subset $E \subseteq \kappa$ for which each element of $E$ is a limit ordinal of cofinality $\omega$ and, for each limit ordinal $\alpha<\kappa$, the set $E \cap \alpha$ is not stationary in $\alpha$.

Proof of Theorem 1.1. Let $\kappa$ be as in the hypotheses of Theorem 1.1. We inductively define a group structure on $\kappa$, and this will serve as our group $G$. Let $\gamma_{0}=\omega$, $\gamma_{\alpha+1}=\gamma_{\alpha}+\gamma_{\alpha}$ and $\gamma_{\beta}=\bigcup_{\alpha<\beta} \gamma_{\alpha}$ for limit ordinal $\beta<\kappa$. The set $\left\{\gamma_{\alpha}\right\}_{\alpha<\kappa}$ is obviously a club set in $\kappa$. We will define a group structure on each $\gamma_{\alpha}$ so that $\gamma_{\alpha}$ will be a subgroup of $\gamma_{\beta}$ whenever $\alpha<\beta$, and thus the group structure on $\kappa=\bigcup_{\alpha<\kappa} \gamma_{\alpha}$ will be well defined.

Select stationary $E_{0} \subseteq \kappa$ satisfying the conclusion of Theorem 3.5 and such that $\kappa \backslash E_{0}$ is stationary in $\kappa$ (using Theorem 3.2). It is easy to check that $E=\left\{\gamma_{\alpha}\right\}_{\alpha \in E_{0}}$ is also stationary in $\kappa$. Let $\left\{\left(T_{\gamma_{\alpha}}^{0}, T_{\gamma_{\alpha}}^{1}\right)\right\}_{\gamma_{\alpha} \in E}$ be a sequence as in Remark 3.4.

The following properties will hold on the groups for all $\alpha<\beta<\kappa$ :

(i) $\gamma_{\beta}$ is free of infinite rank;

(ii) $\gamma_{\alpha}$ is a proper subgroup of $\gamma_{\beta}$;

(iii) $\gamma_{\alpha}$ is a free factor of $\gamma_{\beta}$ if and only if $\gamma_{\alpha} \notin E$;

(iv) $\gamma_{\alpha}^{\prime}=\gamma_{\beta}^{\prime} \cap \gamma_{\alpha}$.

We let $\gamma_{0}$ be free of countably infinite rank. Suppose that we have defined the group structure on $\gamma_{\alpha}$ for all $\alpha<\beta<\kappa$ such that the above conditions hold. If $\beta=\delta+1$ and $\gamma_{\delta} \notin E$, then we give $\gamma_{\beta}$ the group structure obtained by any bijection $f: \gamma_{\beta} \rightarrow \gamma_{\delta} * \mathbb{Z}$ such that $f \uparrow \gamma_{\alpha}$ is the identity map. Such a bijection exists since $\left|\gamma_{\beta} \backslash \gamma_{\delta}\right|=\left|\gamma_{\delta}\right|$. Conditions (i), (ii) and (iv) obviously hold. Notice also that, for $\alpha<\beta$, we have $\gamma_{\alpha}$ a free factor of $\gamma_{\beta}$ if and only if $\gamma_{\alpha}$ is a free factor of $\gamma_{\delta}$ (this uses Lemma 2.1), and so (iii) also holds.

If $\beta=\delta+1$ and $\gamma_{\delta} \in E$, then we consider two subcases. Firstly, suppose that there exists a strictly increasing sequence $\left\{\alpha_{n}\right\}_{n \in \omega}$ for which

- $\gamma_{\alpha_{n}} \notin E$,

- $\cup_{n \in \omega} \alpha_{n}=\delta$,

- $\gamma_{\alpha_{n}}=\left(\gamma_{\alpha_{n}} \cap T_{\gamma_{\delta}}^{0}\right) *\left(\gamma_{\alpha_{n}} \cap T_{\gamma_{\delta}}^{1}\right)$ with both free factors nontrivial for all $n \in \omega$.

From this, it follows immediately that $\gamma_{\delta}=\left(\gamma_{\delta} \cap T_{\gamma_{\delta}}^{0}\right) *\left(\gamma_{\delta} \cap T_{\gamma_{\delta}}^{1}\right)=T_{\gamma_{\delta}}^{0} * T_{\gamma_{\delta}}^{1}$. Since $\left\{\gamma_{\alpha_{n}}\right\}_{n \in \omega}$ is a strictly increasing sequence of sets, we know for each $n \in \omega$ 
that it is either the case that

$$
\gamma_{\alpha_{n+1}} \cap T_{\gamma_{\delta}}^{0} \supsetneq \gamma_{\alpha_{n}} \cap T_{\gamma_{\delta}}^{0} \quad \text { or that } \quad \gamma_{\alpha_{n+1}} \cap T_{\gamma_{\delta}}^{1} \supsetneq \gamma_{\alpha_{n}} \cap T_{\gamma_{\delta}}^{1} .
$$

By choosing a subsequence, we can assume without loss of generality that

$$
\gamma_{\alpha_{n+1}} \cap T_{\gamma_{\delta}}^{1} \supsetneq \gamma_{\alpha_{n}} \cap T_{\gamma_{\delta}}^{1} \quad \text { for all } n \in \omega
$$

(else we exchange the roles of $T_{\gamma_{\delta}}^{0}$ and $T_{\gamma_{\delta}}^{1}$ in the construction that follows). Since each $\gamma_{\alpha_{n}} \notin E$, we know by our induction that $\gamma_{\alpha_{n}}$ is a free factor of $\gamma_{\delta}$ and also of $\gamma_{\alpha_{n+1}}$. Then, by Lemma 2.1, we know that $\gamma_{\alpha_{n}} \cap T_{\gamma_{\delta}}^{1}$ is a free factor of $\gamma_{\delta} \cap T_{\gamma_{\delta}}^{1}$ and of $\gamma_{\alpha_{n+1}} \cap T_{\gamma_{\delta}}^{1}$. Inductively select a free basis $Q$ of $\gamma_{\delta} \cap T_{\gamma_{\delta}}^{1}$ such that $Q \cap \gamma_{\alpha_{n}} \cap T_{\gamma_{\delta}}^{1}$ is a free generating set for $\gamma_{\alpha_{n}} \cap T_{\gamma_{\delta}}^{1}$ for each $n \in \omega$. For each $n \in \omega$, select $t_{n} \in Q \cap \gamma_{\alpha_{n+1}} \cap T_{\gamma_{\delta}}^{1} \backslash \gamma_{\alpha_{n}}$, and let $X=Q \backslash\left\{t_{n}\right\}_{n \in \omega}$. Let $\{y\} \sqcup Y$ be a free generating set for $\gamma_{\delta} \cap T_{\gamma_{\delta}}^{0}$. By how the set $\left\{\gamma_{\alpha}\right\}_{\alpha<\kappa}$ was defined, we know $\left|\gamma_{\beta}\right|=\left|\gamma_{\beta} \backslash \gamma_{\delta}\right|=\left|\gamma_{\delta}\right|$. Hence we let $\gamma_{\beta}$ be given a group structure using a bijection $f: \gamma_{\beta} \rightarrow F(X) * F(\{y\} \cup Y) * F\left(\left\{z_{n}\right\}_{n \in \omega}\right)$ such that $f \uparrow \gamma_{\delta}$ is the isomorphism $\gamma_{\delta} \simeq F(X) * F(\{y\} \cup Y) * F\left(\left\{t_{n}\right\}_{n \in \omega}\right)$ noted above and the inclusion map $\iota: \gamma_{\delta} \rightarrow \gamma_{\beta}$ satisfies $\iota=f^{-1} \circ \phi \circ f \uparrow \gamma_{\delta}$, where $\phi$ is the map from Construction 2.2.

Certainly, conditions (i), (ii) hold, and condition (iv) holds by Lemma 2.3 (iv). Also, we know $\gamma_{\delta}$ is not a free factor of $\gamma_{\beta}$ by Lemma 2.3 (iii). Notice that, for each $n \in \omega$, we have that $\left\langle X \cup\left\{t_{0}, \ldots, t_{n-1}\right\} \cup\{y\} \cup Y\right\rangle$ is a free factor of $\gamma_{\beta}$ by Lemma 2.3 (iii). For each $n \in \omega$, we know $\gamma_{\alpha_{n}}$ is a free factor of

$$
\left\langle X \cup\left\{t_{0}, \ldots, t_{n-1}\right\} \cup\{y\} \cup Y\right\rangle,
$$

and for each $\alpha<\delta$, there exists $n \in \omega$ for which $\alpha<\alpha_{n}$. It follows by Lemma 2.1 and by our induction that, for $\alpha<\delta$, we have $\gamma_{\alpha}$ a free factor of $\gamma_{\beta}$ if and only if $\gamma_{\alpha} \notin E$, and condition (iii) holds.

On the other hand, suppose $\beta=\delta+1$ and $\gamma_{\delta} \in E$ and no such increasing sequence $\left\{\alpha_{n}\right\}_{n \in \omega}$ exists. Since $\delta \in E_{0}$ is of cofinality $\omega$ and $E_{0} \cap \delta$ is not stationary in $\delta$, we may select a strictly increasing sequence $\alpha_{n} \notin E_{0}$ such that $\bigcup_{n \in \omega} \alpha_{n}=\delta$. Then $\gamma_{\alpha_{n}} \notin E$. As each $\gamma_{\alpha_{n}}$ is a free factor of $\gamma_{\delta}$ and the $\alpha_{n}$ are strictly increasing, we may select by induction a free generating set $Q$ for $\gamma_{\delta}$ such that $Q \cap \gamma_{\alpha_{n}}$ is a free generating set for $\gamma_{\alpha_{n}}$. Pick $y \in \gamma_{\alpha_{0}} \cap Q$ and $t_{n} \in \gamma_{\alpha_{n+1}} \cap Q \backslash \gamma_{\alpha_{n}}$, and letting $X=\varnothing$ and $Y=Q \backslash\left(\left\{t_{n}\right\}_{n \in \omega} \cup\{y\}\right)$, we can let $\gamma_{\beta}$ be a group freely generated by $X \cup\left\{z_{n}\right\}_{n \in \omega} \cup\{y\} \cup Y$ such that the inclusion map $\iota: \gamma_{\delta} \rightarrow \gamma_{\beta}$ is the map $\phi$ from Construction 2.2. The check that the induction conditions still hold is as in the other subcase.

When $\beta<\kappa$ is a limit ordinal, the binary operation on $\gamma_{\beta}=\bigcup_{\alpha<\beta} \gamma_{\alpha}$ is defined by that on the $\gamma_{\alpha}$ for $\alpha<\beta$. By how $E_{0}$ was chosen, we know $E_{0} \cap \beta$ is 
not stationary in $\beta$, and so we select a club set $C \subseteq \beta$ for which $C \cap E_{0}=\emptyset$. By induction, we know that, for $\alpha, \delta \in C$ with $\alpha<\delta$, we have that $\gamma_{\alpha}$ is a proper free factor of $\gamma_{\delta}$, and since $C$ is closed, we have $\gamma_{\delta}=\bigcup_{\alpha<\delta, \alpha \in C} \gamma_{\alpha}$ for any $\delta \in C$ which is a limit under the ordering of $\kappa$ restricted to $C$. Then, by induction on $C$, we can select a free generating set $Q$ for $\gamma_{\beta}=\bigcup_{\alpha \in C} \gamma_{\alpha}$ for which $Q \cap \gamma_{\alpha}$ is a free generating set for $\gamma_{\alpha}$ for each $\alpha \in C$. Then (i) and (ii) hold. For $\alpha<\beta$, it is clear by Lemma 2.1 that $\gamma_{\alpha}$ is a free factor of $\gamma_{\beta}$ if and only if $\gamma_{\alpha}$ is a free factor of $\gamma_{\delta}$ for some $\delta \in C$ with $\delta>\alpha$, and since $\gamma_{\delta}$ is a free factor of $\gamma_{\beta}$ for each $\delta \in C$, condition (iii) holds. Condition (iv) follows by induction since $\gamma_{\beta}^{\prime}=\bigcup_{\alpha<\beta} \gamma_{\alpha}^{\prime}$. This completes the construction of the group structure on $\kappa$.

We verify that conditions (1)-(3) of the statement of Theorem 1.1 hold. Imagine for contradiction that $\kappa=A * B$ with $A, B$ nontrivial subgroups of $\kappa$. Letting $\alpha^{\prime}$ be such that $\gamma_{\alpha^{\prime}} \cap A$ and $\gamma_{\alpha^{\prime}} \cap B$ are both nontrivial and

$$
C=\left\{\alpha^{\prime} \leq \alpha<\kappa \mid \gamma_{\alpha}=\left(\gamma_{\alpha} \cap A\right) *\left(\gamma_{\alpha} \cap B\right)\right\},
$$

it is straightforward to verify that $C$ is club in $\kappa$. Since the set $\kappa \backslash E_{0}$ is stationary in $\kappa$, we know $D=C \backslash E_{0}$ is stationary and therefore unbounded in $\kappa$. Then the closure $\bar{D}$ is club in $\kappa$, and so is $\left\{\gamma_{\alpha}\right\}_{\alpha \in \bar{D}}$. Then there exists $\gamma_{\delta} \in E$ with $\delta \in \bar{D}$ and $A \cap \gamma_{\delta}=T_{\gamma_{\delta}}^{0}$ and $B \cap \gamma_{\delta}=T_{\gamma_{\delta}}^{1}$. Then $\delta \in E_{0}$, and so $\delta \in E_{0} \cap \bar{D}$. As $\delta \in E_{0}$, we know that $\delta$ has cofinality $\omega$ in $\kappa$. Certainly, $\delta \notin D=C \backslash E_{0}$, and so there exists a strictly increasing sequence $\left\{\alpha_{n}\right\}_{n \in \omega}$ with $\alpha_{n} \in C \backslash E_{0}$ such that $\bigcup_{n \in \omega} \alpha_{n}=\delta$. Then

$$
\gamma_{\alpha_{n}}=\left(\gamma_{\alpha_{n}} \cap A\right) *\left(\gamma_{\alpha_{n}} \cap B\right)=\left(\gamma_{\alpha_{n}} \cap T_{\gamma_{\delta}}^{0}\right) *\left(\gamma_{\alpha_{n}} \cap T_{\gamma_{\delta}}^{1}\right) .
$$

By our construction, we know that $\gamma_{\delta}$ includes into $\gamma_{\delta+1}$ in such a way that $\gamma_{\delta+1}$ is not a subgroup of $A * B$ (using Lemma 2.4 and letting $(A, B)=\left(L_{0}, L_{1}\right)$ or $(A, B)=\left(L_{1}, L_{0}\right)$ depending on whether we selected $\left\{t_{n}\right\}_{n \in \omega} \subseteq T_{\gamma_{\delta}}^{1}$ or, respectively, $\left\{t_{n}\right\}_{n \in \omega} \subseteq T_{\gamma_{\delta}}^{0}$ in the construction), and this is a contradiction. Thus $\kappa$ is freely indecomposable, and we have part (1).

For part (2), we let $X \subseteq \kappa$ with $|X|<\kappa$. By the regularity of $\kappa$, select $\alpha<\kappa$ large enough that $\gamma_{\alpha} \supseteq X$. Notice that $\gamma_{\alpha+1} \notin E$. Any subgroup $H$ of $\kappa$ with $|H|<\kappa$ and $H \geq \gamma_{\alpha+1}$ satisfies $H \leq \gamma_{\beta}$ for some $\beta>\alpha$ by the regularity of $\kappa$. Since $\gamma_{\alpha+1}$ is a free factor of $\gamma_{\beta}$ by our construction, we have by Lemma 2.1 that $\gamma_{\alpha+1}$ is a free factor of $H$. Thus $\gamma_{\alpha+1}$ is $\kappa$-pure, and the group $\kappa$ is strongly $\kappa$-free, and we have verified (2).

For part (3), we notice that, since

$$
\gamma_{\alpha}^{\prime}=\gamma_{\alpha} \cap \gamma_{\beta}^{\prime} \quad \text { for each } \alpha<\beta, \quad \text { and since } \quad \kappa^{\prime}=\bigcup_{\beta<\kappa} \gamma_{\beta}^{\prime},
$$


the equality $\gamma_{\alpha}^{\prime}=\gamma_{\alpha} \cap \kappa^{\prime}$ holds for all $\alpha<\kappa$. In particular, the homomorphism induced by the inclusion map $\gamma_{\alpha} / \gamma_{\alpha}^{\prime} \rightarrow \kappa / \kappa^{\prime}$ is injective, and so the abelianization of $\kappa$ is the increasing union of free abelian subgroups $\gamma_{\alpha} / \gamma_{\alpha}^{\prime}$. In our construction, when $\beta=\delta+1$ and $\gamma_{\delta} \in E$, the map induced by inclusion $\gamma_{\delta} / \gamma_{\delta}^{\prime} \rightarrow \gamma_{\beta} / \gamma_{\beta}^{\prime}$ is an isomorphism by Lemma 2.3 (iv). When $\beta=\delta+1$ and $\gamma_{\delta} \notin E$, we have $\gamma_{\delta} / \gamma_{\delta}^{\prime}$ as a proper direct summand of $\gamma_{\beta} / \gamma_{\beta}^{\prime}$. As well, we have $\gamma_{\beta} / \gamma_{\beta}^{\prime}=\bigcup_{\alpha<\beta} \gamma_{\alpha} / \gamma_{\alpha}^{\prime}$ for limit $\beta<\kappa$. Thus we may inductively select a free abelian basis for the abelianization of $\kappa$, and the free basis will be of cardinality $\kappa$ since there are $\kappa$ many $\beta$ for which $\beta=\delta+1$ with $\gamma_{\delta} \notin E$. We have verified (3) and finished the proof of the theorem.

Acknowledgments. The author thanks the anonymous referee for their careful reading and suggested improvements which enhanced the presentation of the paper.

\section{Bibliography}

[1] C. Bitton, The abelianization of almost free groups, Proc. Amer. Math. Soc. 129 (2001), no. 6, 1799-1803.

[2] P. C. Eklof, On the existence of $\kappa$-free abelian groups, Proc. Amer. Math. Soc. 47 (1975), 65-72.

[3] P. C. Eklof, Methods of logic in abelian group theory, in: Abelian Group Theory, Lecture Notes in Math. 616, Springer, Berlin (1977), 251-269.

[4] P. C. Eklof and A. H. Mekler, On constructing indecomposable groups in L, J. Algebra 49 (1977), no. 1, 96-103.

[5] P. C. Eklof and A. H. Mekler, Almost Free Modules. Set-theoretic Methods, NorthHolland Math. Libr. 46, North-Holland, Amsterdam, 1990.

[6] K. Gödel, The consistency of the axiom of choice and of the generalized continuum hypothesis, Proc. Natl. Acad. Sci. USA 24 (1938), 556-557.

[7] G. Higman, Almost free groups, Proc. Lond. Math. Soc. (3) 1 (1951), 284-290.

[8] G. Higman, Some countably free groups, in: Group Theory, De Gruyter, Berlin (1989), 129-150.

[9] T. Jech, Set Theory: The Third Millenium Edition, Springer Monogr. Math., Springer, Berlin, 2006.

[10] R. B. Jensen, The fine structure of the constructible hierarchy, Ann. Math. Logic 4 (1972), 229-308.

[11] R. C. Lyndon and P. E. Schupp, Combinatorial Group Theory, Ergeb. Math. Grenzgeb. (3) 89, Springer, Berlin, 1977, 
[12] M. Magidor and S. Shelah, When does almost free imply free? (For groups, transversals, etc.), J. Amer. Math. Soc. 7 (1994), no. 4, 769-830.

[13] A. H. Mekler, How to construct almost free groups, Canadian J. Math. 32 (1980), no. $5,1206-1228$.

[14] T. Nishinaka, Uncountable locally free groups and their group rings, J. Group Theory 21 (2018), no. 1, 101-105.

[15] S. Shelah, A compactness theorem for singular cardinals, free algebras, Whitehead problem and transversals, Israel J. Math. 21 (1975), no. 4, 319-349.

[16] R. M. Solovay, Real-valued measurable cardinals, in: Axiomatic Set Theory, Proc. Sympos. Pure Math. 13 Part 1, American Mathematical Society, Providence (1971), 397-428.

Received July 15, 2019; revised November 25, 2019.

\section{Author information}

Samuel M. Corson, Instituto de Ciencias Matemáticas CSIC-UAM-UC3M-UCM, 28049 Madrid, Spain.

E-mail: sammyc973@gmail.com 